\title{
Toy Languages and Free Imaginations
}

\author{
Katuzi ONo
}

\section{Introduction}

Our real mathematical thinking is really complicated. Surely, we have simple mathematical theories developable from their respective axiom or postulate systems in a certain restricted language, a language without tense and modality, and being fortified by successive definitions of notions starting from a definite number of primitive notions. However, in our real mathematical thinking, íe apply mathematics to mathematics. For example, we apply group theory to simple geometry which is considered to be developable from pure geometrical axioms. To understand our real activity correctly, we have to understand our language for developing simple mathematical theories as well as our language for applying mathematics to mathematics. Even the language for developing simple mathematical theories can never be very simple.

Even in simple mathematical theories, we usually deal with objects consisting of an infinite number of objects. However, this kind of things must be products of our imagination. We have to think of some imaginative principles hidden in our language for simple mathematical theories which leads us to the kind of things.

We can not believe that our language activity is so fullfledged from the beginning. Our language even for simple theories of mathematics must have been brought up in the course of our thinking.

It is hard to get a clean cut view of our real language. As an approach to better understanding of our real language, we imagine here a simple toy language of which we can get a clean cut view, and we consider how we would bring it up to a fullfledged toy language by our free daring imaginations.

The basic idea of this paper is that the following tendency of us is very important for understanding our imaginative activity:

Whenever we admit any proposition, we are apt to imagine a mini-universe where the proposition holds.

\section{Real mood of our thinking}

We feel ourselves often thinking, thinking in our ordinary language. In a special mood of thinking, we reason mathematically in a special part of our ordinary language, a tenseless part. Free imaginations motivated by knowing real aspect of deductions in our ordinary language seem to built up the whole structure of our mathematical reasonings.

Considerations of this paragraph are along the line [1]. 
1.1. In our communication, speakers seem to be in the mood that they have something in their minds and express the something by sentences, and hearers seem to be in the mood that they know the something in the speakers minds by the sentences they heard.

1.1.1. Presumptuous or not, we are in similar mood also in our mathematical reasonings.

1.1.2. Our ordinary language for mathematical reasonings has been fortified by introducing various expressions, object variables, etc., and will be further fortifed by devices still unknown to us.

1.1.3. Presumptuous or not, we are in the mood of having the sentence notion and being able to express any proposition in our minds through a sentence.

1.1.4. Presumptuous or not, we are in the mood of being able to imagine propositions by sentences.

1.1.5. Presumptuous or not, we are in the mood of being able to express propositions by sentences of a normalized style, may be admitted by a special grammer. Choice of the normalized style must be a matter of taste to some extent.

1.2. Presumptuous or not, we are in the mood of having the deduction notion.

1.2.1. Deductions seem to be personal. Everyone would have his own skipping deductions in his mind.

1.2.2. We are apt to think of deductions common to all, maybe obtainable by refining personal skipping deductions.

1.2.3. Presumptuous or not, we are in the mood of being able to express fine deductions in a standard style. Choice of the standard style of fine deductions is surely a matter of tast to some extent.

1.3. In our resoning, we are in the mood to use notions only after defining them. In reality, we must admit a primitive vocabulary, the set of primitive notions, from the beginning.

1.3.1. In our real reasoning, we are not conscious of the primitive vocabulary exactly.

1.3.2. We are in the mood to define notions one after another in a series.

1.3.3. We are in the mood to define any notion in terms of notions in the primitive vocabulary and the notions defined beforehand.

1.4. We are in the mood to compose sentences in terms of notions.

1.4.1. In our real reasoning, we are not conscious of tools for composing sentences in our ordinary language.

1.4.2. The tools for composing sentences in our ordinary language seem to play essential roles for our reasoning activity.

1.4.3. We use free and bound variables for composing sentences in our fortified ordinary language. We tacitly disregard nominal distinction of bound variables 
between composed sentences in the fortified language.

1.5. We are in the mood to define any notion by a condition to be imposed on a definite number of variables.

1.5.1. Any primitive notion might be applicable to various number of objects, but each notion thus defined is applicable only to a definite number of objects.

1.6. We introduce any condition to be imposed on a definite number of objects by stating a sentence with respect to the same number of variables taken up for the purpose of defining the condition.

1.6.1. Between definitions of conditions, we tacitly disregard nominal distinction of variables taken up for the purpose of defining the conditions.

1.7. Fallacious or not, we are strongly tempted to regard any notion as an object.

1.8. We are apt to consider any sentence in a certain range of objects.

1.8.1. Whenever we admit any sentence, we are strongly tempted to imagine a mini-universe where the sentence holds.

\section{A special style for describing fine deductions.}

To meet practical need, we introduce here a special style for describing fine deductions.

The style is essentially the same as the style introduced in [2].

2.1. Our practical style.

We have endeavored to establish a style in which the formal structure of fine deductions would be exhibited perspectively.

2.1.1. We understand any fine deduction as a series of steps.

2.1.2. We understand any step of a fine deduction either as an assumption step introducing some free variables and stating some assumptions with respect to them, or as an assertion step.

2.1.3. It is our policy to give an index to each step for reference's sake.

2.1.4. Any step has its proper available range clearly shown by indices.

2.1.5. Any step is qualified to be a basis of every assertion step in its available range.

2.1.6. Any assertion step should be either deduced directly from some foregoing steps qualified to be its bases, or should be proved by a partial deduction following just after it.

\subsection{Index system}

It is our policy to denote indices by finite series of letters $A, b, \cdots, z, \cdots, \#$.

2.2.1. We regard \# as a small letter.

2.2.2. We may supply small letters for indices between any pair of letters for 
indices as occasion calls.

2.2.3. For communication' sake, we use small Greek letters to denote finite series of small letters for indices including the length 0 case.

2.3. Rules for our reference system.

2.3.1. We spell the index of any assertion step in the form $\sigma$.

2.3.2. We spell the index of any assumption step in the form $\sigma A$.

2.3.3. We introduce any assertion step directly deducible from its foregoing step in the form $\sigma$ ).

2.3.4. We introduce any assertion step to be proved by a partial deduction following just after it in the form $\sigma$ )).

2.3.5. We refer to the step introduced in the form $\sigma$ ) as well as in the form $\sigma$ )) simply by $o$.

2.3.6. Any step is in the available range of an assumption step $\alpha A$ if and only if its index is of the form $\sigma p r$.

2.3.7. Any step is in the available range of an assertion step op if and only if the index of the step is of the form $\sigma q \tau$ for a small letter $q$ standing after $p$ in the letter series for indices.

2.3.8. The partial deduction for proving an assertion step $\sigma$ consists of all the steps of the forms $\sigma \tau \mathrm{A}$ and $\sigma \tau \mathrm{p}$. For any step of the form $\sigma \rho p$, there must be steps of the forms $\sigma \rho \mathrm{A}$ and $\sigma \rho \neq$ in the partial deduction.

2.4. In any assertion step, we place one and only one stentence.

2.5. Forms of assumption steps.

General form of assumption step is

oA) Take any arbitrary $x, \cdots, y$ such that

1) $A, \cdots$, n) $\mathscr{B}$.

Formally,

oA) $x, \cdots, y$

1) $A, \cdots, \mathrm{n}) \mathscr{B} . \quad(\mathrm{n}=0,1, \cdots)$

2.5.1. The special case of the form

øA) Assume

1) $A, \cdots, n) \mathscr{B}$.

is formally described in the form

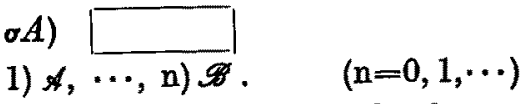

2.5.2. The special case of the form

oA) Take any arbitray $\boldsymbol{x}, \cdots, \boldsymbol{y}$.

is formally described in the form

oA) $x, \cdots, y$.

2.5.3. The special case of the form 
oA) Without any assumption

is formally interpreted in the form

øA)

2.6. The phrase "any arbitrary $x, \cdots, \boldsymbol{y}$ " in any assumption step oA of any fine deduction can be agreeably interpreted into the conditions

(1) $\boldsymbol{x}, \cdots, \boldsymbol{y}$ is a non-repetitive series of free variables,

(2) $\boldsymbol{x}, \cdots, \boldsymbol{y}$ do not occur in any step qualified to be a basis of the step $\sigma$ or in the step $\sigma$.

2.6.1. For any expression of the form $\mid \overrightarrow{x, \cdots, y}$ in any assumption step of the form $\sigma A$, we tacitly assume that $x, \cdots, y$ satisfy the above conditions (1) and (2).

2.7. Remarks

2.7.1. There may be some comments in our ordinary language for any fine deduction described in our special style for easier understanding.

2.7.2. We may describe formal deductions skippingly assuming that we are confident of fulfilling the omitted steps as occasion calls.

2.7.3. We call any pair of expressions to be logically equivalent if it is confirmed that we have a right skipping deduction when we replace any one of the pair in any right deduction by the other.

\section{Toy languages}

Reasonable or not, we are apt to believe in numerical computations or judgements by rules. We would like to manufacture toy languages each having clear rules for compositions of toy sentences and deductions of toy sentences from series of toy sentences.

\subsection{Toward toy languages}

We are unconsciously thinking in our ordinary language. This is the case even in our mathematical reasonings.

3.1.1. Our ordinary language is really full of life, pregnant, and too universal for our mathematical reasonings.

3.1.2. The language really used in our mathematical reasonings is a fortified language of our ordinary language by introducing object variables, special symbols, and various expressions.

3.1.3. The language used in our mathematical reasonings is a special part of our ordinary language independent of tenses and modalities.

3.1.4. We would like to copy the essentials of our ordinary language really used in our mathematical reasonings by toy languages going to be manufactured.

3.2. Expressions in our toy languages

Presumptuous or not, we are confident of discerning certain kinds of symbols such as letters, numerals, etc., and certain kinds of expressions, usually configura- 
tions of symbols without falling into errors.

3.2.1. Object variables in the fortified part of our ordinary language are used either as free or bound.

3.2.2. In the fortified part of our ordinary language, the same kind of letters are usually used for denoting both kinds of object variables.

3.2.3. Presumptuous or not, we are confident of discerning free and bound variables by contexts in our ordinary language.

3.2.4. Presumptuous or not, we are confident of dealing with finite series of of expressions. For communication, let us establish the following convention:

When we speak of expression series of any length, we tacitly assume that they are series of finite lengths including the length 0 case.

3.2.5. In the following, we denote expressions in general by notations of the form " $\theta$ ".

3.3. Object variables in our toy languages

As materials of our toy languages going to be manufactured, we are intending to use two kinds of object variables distinctly in toy sentences and toy conditions.

3.3.1. We use small Gothic letters such as $x, y, \cdots$ for free variables and small bold face letters such as $\mathbf{x}, \mathbf{y}, \cdots$ for bound variables.

3.3.2. For communication, we use small italic letters such as $x, y, \cdots$ for representing free variables as well as bound variables.

\subsection{Variable change}

Presumptuous or not, we are confident of our ability to change object variables of expressions into other object variables without falling into errors.

3.4.1. Let $x, \cdots, y$ be any non-repetitive series and $u, \cdots, v$ be any series of the same length. Then, we use variable change notations of the form $\left(\begin{array}{l}x, \cdots, y \\ u, \cdots, v\end{array}\right)$ for communication for the purpose of representing the expression obtained by changing $x, \cdots, y$ of any expression " $\theta$ " into $u, \cdots, v$, respectively, by $\left(\begin{array}{c}x, \cdots, y \\ u, \cdots, v\end{array}\right)$ (" $\theta$ ").

3.4.2. Whenever we use any variable change notation of the form $\left(\begin{array}{l}x, \cdots, y \\ u, \cdots, v\end{array}\right)$, we assume tacitly

(1) $x, \cdots, y$ is a non-repetitive series of the length $\mathrm{k}$,

(2) $u, \cdots, v$ is a series of the length $\mathrm{k}$

for some $\mathrm{k}(=0,1, \cdots)$.

We call $\left(\begin{array}{l}x, \cdots, y \\ u, \cdots, v\end{array}\right)$ a variable change of $\mathrm{k}$ variables.

3.5. On primitive notions and primitive vocabularies

In our ordinary language, we are really assuming to have some primitive notions. However, our primitive vocabulary i.e. the set of all the primitive. 
notions seems to be ever increasing, so the concept of the primitive vocabulary is quite ambiguous. We can only assume that our primitive vocabulary is sufficiently rich.

3.5.1. In our ordinary language, we have surely such a notion that is applicable only to a definite number of objects, but we have also such a notion that is applicable to any number of objects.

3.5.2. To be general, we assume that each primitive notion is applicable to any number of objects.

3.5.3. For any series $x, \cdots, y$ of any length and any primitive notion $P$, wer egard any expression of the form $x \cdots y \mathrm{P}$ as a toy sentence.

\subsection{Composition tools}

Most of mathematical theorems can be described in the general implication form

Any $x, \cdots, y$ satisfying the conditions $F, \cdots, G$ satisfy the condition $H$.

We regard the general implication as the only composition tool of our basic toy language.

3.6.1. In the following, we are intending to denote toy sentences by capital script letters.

3.6.2. We express the general implication in the toy sentence form

$$
\forall \mathbf{x} \cdots \mathbf{y}\left(\begin{array}{c}
x, \cdots, \boldsymbol{y} \\
\mathbf{x}, \cdots, \mathbf{y}
\end{array}\right)\left(\frac{\mathscr{F}, \cdots, \mathscr{E}}{\mathscr{H}}\right)
$$

in our basic toy language, where $\mathscr{F}, \cdots, \mathscr{G}, \mathcal{K}$ is assumed to be series of any length no shorter than 1 and $\mathbf{x}, \cdots, \mathbf{y}$ is assumed to be a non-repetitive series containing no term occring in $\mathscr{F}, \cdots, \mathscr{F}, \mathcal{H}$.

\subsection{Toy conditions}

In our ordinary language, we introduce conditions by stating sentences with respect to a number of variables for defining conditions.

3.7.1. In our basic toy languages, we define any toy condition by stating a toy sentence $\mathscr{F}$ with respect to some variables $x, \cdots, y$ for defining the condition and we express the toy condition on these variables by

$$
\left[\mathbf{x}, \cdots, \mathbf{y} /\left(\begin{array}{l}
x, \cdots, y \\
\mathbf{x}, \cdots, \mathbf{y}
\end{array}\right)(\mathscr{F})\right]
$$

where the series $\mathbf{x}, \cdots, \mathbf{y}$ is assumed to be non-repetitive and to have no term occurring in $\mathscr{F}$.

3.7.2. We call $\left[\mathbf{x}, \cdots, \mathbf{y} /\left(\begin{array}{l}x, \cdots, y \\ \mathbf{x}, \cdots, y\end{array}\right)(\mathscr{F})\right]$ for any non-repetitive series $\mathbf{x}, \cdots, y$ of the length $\mathrm{k} a$ toy condition on $\mathrm{k}$ variables. $(\mathrm{k}=0,1, \cdots)$

3.7.3. Let $u, \cdots, v$ be any series of the length $k$ and $\left[x, \cdots, y /\left(\begin{array}{l}x, \cdots, y \\ x, \cdots, y\end{array}\right)(\mathscr{F})\right]$ be 
any toy condition on $\mathrm{k}$ variables. Then, $u \cdots v\left[, \mathbf{x} \cdots, \mathbf{y} /\left(\begin{array}{l}x, \cdots, \boldsymbol{y} \\ \mathbf{x}, \cdots, \mathbf{y}\end{array}\right)(\mathscr{F})\right]$ denotes the toy sentence $\left(\begin{array}{l}x, \cdots, y \\ u, \cdots, v\end{array}\right)(\mathscr{F})$ which means that $u, \cdots, v$ satisfy the toy condition $\left[\mathbf{x}, \cdots, \mathrm{y} /\left(\begin{array}{l}x, \cdots y \\ \mathbf{x}, \cdots, y\end{array}\right)(\mathscr{F})\right] . \quad(\mathrm{k}=0,1, \cdots)$

3.7.4. In the following, we are intending to denote any toy condition on $k$ variables in the form $[k ! \mathscr{C}]$ for any capital script type letter $\mathscr{C} .(k=0,1, \cdots)$

4. Clearly stated rules for toy sentence compositions and toy deductions in basic toy languages

Toy languages are worthy to be created as long as their rules for toy sentence compositions and toy deductions can be stated clearly.

Logical rules of basic toy languages introduced here bear a close resemblance to the primitive logic introduced in [3].

4.1. Rules for composing toy sentences and toy conditions

4.1.1. Any expression of the form $\boldsymbol{x}, \cdots, \boldsymbol{y P}$ for any series $\boldsymbol{x}, \cdots, \boldsymbol{y}$ of any length and any primitive notion $\mathbf{P}$ is a toy sentence.

We call toy sentences of this kind elementary.

4.1.2. Let $\mathscr{I}, \cdots, \mathscr{S}, \mathcal{H}$ be any toy sentence series of any length no shorter than 1 , $\left(\begin{array}{l}x, \cdots, y \\ \mathbf{x}, \cdots, \mathbf{y}\end{array}\right)$ be any variable change such that $\mathbf{x}, \cdots, \mathbf{y}$ do not occur in $\mathscr{F}, \cdots, \mathscr{C}, \mathcal{H}$, and $\frac{\mathscr{F}^{*}, \cdots, \mathscr{G}^{*}}{\mathcal{H}^{*}}$ be the expression $\left(\begin{array}{l}\boldsymbol{x}, \cdots, \boldsymbol{y} \\ \mathbf{x}, \cdots, \mathbf{y}\end{array}\right)\left(\frac{\mathscr{F}, \cdots, \mathscr{F}}{\mathcal{H}}\right)$. Then, $\forall \mathbf{x} \cdots \mathbf{y} \frac{\mathscr{F}^{*}, \cdots, \mathscr{C}^{*}}{\mathcal{H}^{*}}$ is a toy sentence.

4.1.3. Let $F$ be any toy sentence, $\left(\begin{array}{l}x, \cdots, y \\ \mathbf{x}, \cdots, y\end{array}\right)$ be any change of $\mathrm{k}$ variables such that $\mathbf{x}, \cdots, \mathbf{y}$ do not occur in $\mathscr{F}$, and $\mathscr{F}^{*}$ be the expression $\left(\begin{array}{l}x, \cdots, y \\ \mathbf{x}, \cdots, \mathbf{y}\end{array}\right)(\mathscr{F})$. Then, the expression $\left[\mathbf{x}, \cdots, \mathbf{y} / \mathscr{F}^{*}\right]$ is a toy condition on $\mathrm{k}$ variables. $\quad(\mathrm{k}=0,1, \cdots)$

4.1.4. Let $[\mathrm{k} ! \mathscr{E}]$ be a condition of the form $[\mathrm{x}, \cdots, \mathrm{y} / \mathscr{F}]$ such that $\mathrm{x}, \cdots, \mathrm{y}$ do not occur in $F$, and $u, \cdots, v$ be any series of the length $k$. Then, $u \cdots v[k ! \mathscr{E}]$ represents the toy sentence $\left(\begin{array}{l}\boldsymbol{x}, \cdots, \boldsymbol{y} \\ \boldsymbol{u}, \cdots, \boldsymbol{v}\end{array}\right)(\mathscr{F}) . \quad(\mathrm{k}=0,1, \cdots)$

4.2. We can see that any toy sentence of any basic toy language can be expressed without making use of toy condition notations.

4.3. We have nicely formalized predicate logic for our use. To be faithful to our real reasoning, however, we have to modulate the usual predicate logic a little. 
4.3.1. We modulate the usual predicate logic so that we can admit the fact that any proposition independent of $x$ denotes the same thing as that the proposition holds for every $x$.

4.4. Now, we state the rules for toy deductions of basic languages described in our special style introduced in 2.

4.4.1. Let $\mathscr{F}, \ldots, \mathscr{G}, \mathcal{H}$ be any sentence series of any length no shorter than $1 . \quad$ Then, $\mathcal{H}$ is called to be deducible from $\mathscr{F}, \ldots, \mathscr{S}$ if and only if we have a toy deduction beginning with a step of the form

1)) $\forall \mathbf{a} \frac{\mathscr{F}, \cdots, \mathscr{E}}{\mathcal{H}}$

for any a not occurring in $\mathscr{F}, \ldots, \mathscr{E}, \mathcal{H}$.

4.4.2. For any step of the form

-A)) $A$,

A must be expressible in the form

$$
\forall \mathbf{x} \cdots \mathbf{y} \frac{\mathbf{x} \cdots \mathbf{y}[\mathrm{k} ! \mathscr{F}], \cdots, \mathbf{x} \cdots \mathbf{y}[\mathrm{k} ! \mathscr{E}]}{\mathbf{x} \cdots \mathbf{y}[\mathrm{k} ! \mathcal{H}]}
$$

for a non-repetitive series $\mathbf{x}, \cdots, \mathbf{y}$ of the length $\mathbf{k}$ and having no term occurring in the toy condition series $[k ! \mathscr{F}], \cdots,[k ! \mathscr{E}],[k ! \mathscr{K}]$ of the length no shorter than 1 , and there must be stepes expressible in the forms

oA) $u, \cdots, v$

and
1) $u \cdots v[\mathrm{k} ! g], \quad \cdots$,
n) $u \cdots v[\mathrm{k} ! \mathscr{E}]$

o甘) $u \cdots v[\mathrm{k} ! \mathcal{K}], \quad$ or, $\left.\left.\sigma_{\sharp}^{\sharp}\right)\right) \quad u \cdots v[\mathrm{k} ! \mathcal{K}] \quad(\mathrm{k}=0,1, \cdots ; \mathrm{n}=0,1, \cdots)$

4.4.3. For any step of the form

o) $A$

\& must be a toy sentence expressible in the form $\boldsymbol{u} \cdots v[\mathrm{k} ! \mathcal{H}]$, and there must be toy sentences of the forms

$$
\forall \mathbf{x} \cdots \mathbf{y} \frac{\mathbf{x} \cdots \mathbf{y}[\mathrm{k} ! \mathscr{F}], \cdots, \mathbf{x} \cdots \mathrm{y}[\mathrm{k} ! \mathscr{E}]}{\mathrm{x} \cdot \mathrm{y}[\mathrm{k} ! \mathcal{H}]}, \boldsymbol{u} \cdots v[\mathrm{k} ! \mathscr{F}], \cdots, \quad u \cdots v[\mathrm{k} ! \mathscr{E}]
$$

in some steps qualified to be bases of the step $\sigma$, where $\mathbf{x}, \cdots, \mathbf{y}$ are assumed not to occur in $[\mathrm{k} ! \mathscr{F}], \cdots,[\mathrm{k} ! \mathscr{E}],[\mathrm{k} ! \mathcal{H}]$.

4.5. We call any toy sentence deducible if and only if it is deducible from no assumption, i.e. from toy sentence series of the length 0 .

4.6. When we use bound variables in our ordinary language, we disregard nominal distinction of bound variables between toy sentences as well as between toy conditions.

In any basic toy language, we call toy sentences and toy conditions differing only in nominations of bound variables to be similar. 
Concepts of similar toy sentences and similar toy conditions are really important for our toy languages, because these concepts lead us to a big jump from toy sentences and toy conditions to toy propositions and toy notions, respectively. We introduce here these concepts exactly in basic toy language by the following rules: (4.6.1-4.6.4)

4.6.1. Any toy sentence $\mathscr{F}$ of the form $x \cdots y \mathrm{P}$ for any primitive notion $\mathrm{P}$ is similar to any toy sentence $\mathscr{S}$ if and only if $\mathscr{G}$ coincides with $\mathscr{F}$.

4.6.2. Any toy sentences of of the form $\forall \mathbf{x} \cdots \mathbf{y}\left(\left(\begin{array}{l}x, \cdots, \boldsymbol{y} \\ \mathbf{x}, \cdots, y\end{array}\right)\left(\frac{\mathscr{F}, \cdots, \mathscr{G}}{\mathcal{H}}\right)\right)$ is similar to another toy sentence $\mathscr{A}^{*}$ if and only if $\mathscr{A}^{*}$ is a toy sentence of the form

$\forall \mathbf{u} \cdots \mathbf{v}\left(\left(\begin{array}{l}\boldsymbol{x}, \cdots, \boldsymbol{y} \\ \mathbf{u}, \cdots, \mathbf{v}\end{array}\right)\left(\frac{\mathscr{F}^{*}, \cdots, \mathscr{G}^{*}}{\mathcal{H}^{*}}\right)\right)$ such that $\mathscr{F}^{*}, \cdots, \mathscr{G}^{*}$, and $\mathcal{H}^{*}$ are toy sentcnes similar to $\mathscr{F}, \cdots, \mathscr{E}$, and $\mathcal{H}$, respectively.

4.6.3. Any toy condition $[\mathrm{k} ! \mathscr{E}]$ of the form $\left[\mathbf{x}, \cdots, \mathrm{y} /\left(\begin{array}{l}x, \cdots, y \\ \mathbf{x}, \cdots, y\end{array}\right)(\mathscr{F})\right]$ is similar to any toy condition $\left[\mathrm{k} ! \mathscr{C}^{*}\right]$ if and only if $\left[\mathrm{k} ! \mathscr{C}^{*}\right]$ is a toy condition of the form $\left[\mathbf{u}, \cdots, \mathbf{v} /\left(\begin{array}{l}x, \cdots, \boldsymbol{y} \\ \mathbf{u}, \cdots, \mathbf{v}\end{array}\right)\left(\mathscr{F}^{*}\right)\right]$ such that $\mathscr{F}^{*}$ is a toy sentence similar to $\mathscr{F}$.

4.6.4. Any toy sentence $A$ of the form $\mathrm{s} \cdots t[\mathrm{k} ! \mathscr{E}]$ is similar to any toy sentence $A^{*}$ if and only if $\mathscr{A}^{*}$ is a toy sentence of the form $\mathrm{s} \cdots t\left[\mathrm{k} ! \mathscr{C}^{*}\right]$ such that $\left[\mathrm{k} ! \mathscr{C}^{*}\right]$ is similar to $[\mathrm{k} ! \mathscr{E}]$.

4.7. In any basic toy language,

(1) Similar toy sentences are logically equivalent.

(2) Similar toy conditions are logically equivalent.

4.7.1. It should be remarked here that similar toy sentences are deducible from each other only when they are not reducible to elementary toy sentences.

4.7.2. Any free variable occurring in any toy sentence occurs in every toy sentence similar to it.

Any toy condition similar to a toy condition of the form $[\mathrm{k} ! \mathscr{E}]$ is a toy condition of the form $[\mathrm{k} ! \mathscr{D}] .(\mathbf{k}=0,1, \cdots)$

Any free variable occurring in any toy condition occurs in every toy condition similar to it.

\section{Toy propositions and toy notions}

In any basic toy language, we deal with toy sentences and toy conditions. According to 4.7, we can deal with them in any toy deduction by bundles of similar toy sentences and bundles of similar toy conditions. 
Presumptuous or not; we dare to imagine toy boxes to encase - similar toy sentences and similar toy conditions in each.

We call any toy box for encasing similar toy sentences a toy proposition, and any toy box for encasing similar toy conditions a toy notion. Toy propositions and toy notions altogether are toy boxes.

5.1. Deductions with respect to toy boxes.

In our real thinking, we describe any deduction by sentences, but we are in the mood of dealing with propositions represented by the sentences. Accordingly, any conclusion described by a sentence is regarded as a conclusion of the proposition represented by the sentence.

Also in our toy deduction of basic toy language, we regard any toy sentence in a step of any toy deduction as representing a toy proposition and we regard any toy deduction as deduction of a toy proposition from toy proposition series. In other words, we disregard distinction between similar toy sentences and distinction between similar toy conditions.

5.1.1. If any free variable $\boldsymbol{x}$ occurs in any sample of a toy box, $\boldsymbol{x}$ occurs in every sample of the toy box (cf. 4.7.2.). Accordingly, we can say that any free variable $\boldsymbol{x}$ occurs in a toy box. In this case, we call $\boldsymbol{x}$ a free parameter of the toy box.

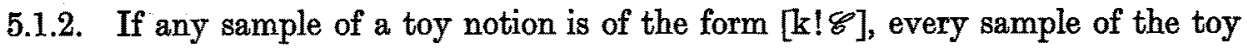
notion must be a condition of the same form. (cf. 4.7.2.) Accordingly, we can say that any toy notion is a $\mathrm{k}$ variable toy notion if and only if samples of the toy notion are of the form $[k ! \mathscr{C}] .(k=0,1, \cdots)$

\subsection{Labels of toy boxes}

We would like to put a label on each toy box. Any sample of any toy box would work well as its label. As for labels, however, the simpler the better. To be practical, we introduce here a special system of labels for toy boxes which bear a close resemblance to sentences and conditions in our ordinary language.

Now, we introduce labels for toy boxes. To match with our ordinary language, we use small italic letters $x, y, \cdots$ only for object variables in labels of toy boxes.

Presumptuous or not, we assume that we can use small italic letters preserving one to one correspondence to small Gothic letters.

5.2.1. In any basic toy language, we use expressions of the form $x \cdots y \mathrm{P}$ for any primitive notion $\mathrm{P}$ as labels of the toy proposition represented by the toy sentence $\boldsymbol{x} \cdot \boldsymbol{y} \mathbf{p}$.

5.2.2. We use expressions of the form $\forall x \cdots y \frac{F, \cdots, G}{H}$ for any labels $F, \cdots, G$, $H$ of the toy propositions $\mathbf{F}, \cdots, \mathbf{G}, \mathbf{H}$, respectively, as labels of the toy proposition represented by toy sentences of the form $\forall \mathbf{x} \cdots \mathbf{y}\left(\left(\begin{array}{c}x, \cdots, y \\ x_{3}, \cdots, y\end{array}\right)\left(\frac{\mathscr{F}, \cdots, \mathscr{E}}{\mathcal{H}}\right)\right)$ for any 
samples $\mathscr{F}, \cdots, \mathscr{B}, \mathcal{H}$ of the toy propositions $\mathbf{F}, \cdots, \mathbf{G}, \mathbf{H}$, respectively.

We denote the toy proposition with labels of the form $\forall x \cdots y \frac{F, \cdots, G}{H}$ simply by $\forall x \cdots y \frac{\mathbf{F}, \cdots, \mathbf{G}}{\mathbf{H}}$.

5.2.3. We use expressions of the forms $[x, \cdots, y / F]$ for any label $F$ of a toy proposition $\mathbf{F}$ as labels of the toy notion represented by the sample toy condition $\left[\mathbf{x}, \cdots, \mathbf{y} /\left(\begin{array}{l}x, \cdots, y \\ \mathbf{x}, \cdots, \mathbf{y}\end{array}\right)(\mathscr{F})\right]$.

We express labels of $\mathrm{k}$ variable toy notions in general by symbols of the form $[\mathrm{k} ! N]$ and $\mathrm{k}$ variable toy notions themselves by symbols of the form [k!N].

We denote the toy notion with labels of the form $[x, \cdots, y / F]$ simply by $[x, \cdots, y / \mathbf{F}]$.

5.2.4. We use expressions of the form $x \cdots y[\mathrm{k} ! N]$ for any label $[\mathrm{k} ! N]$ of a toy notion $[\mathrm{k} ! \mathrm{N}]$ as labels of the toy proposition represented by toy sentences of the form $\boldsymbol{x} \cdot \boldsymbol{y}[\mathrm{k} ! \mathrm{N}]$ for a sample toy condition $[\mathrm{k} ! \mathscr{N}]$ of the toy notion $[\mathrm{k} ! \mathbf{N}]$.

We express the toy proposition with the label $x \cdots y[\mathrm{k} ! N]$ by $x \cdots y[\mathrm{k} ! \mathrm{N}]$.

5.2.5. We call any occurrence of $x$ in any label $A$ of a toy box $A$ free or bound according as it corresponds to a free or bound variable in any sample toy sentence $A$ of A, respectively.

5.2.6. Let $A$ be any label of a toy box $\mathbf{A}$. Then, any sample of the toy box $\mathbf{A}$ is also called a sample of the label $A$.

5.3. Composition rules of labels

We call labels of toy propositions and toy notions label sentences and label conditions, respectively.

The composition rules of label sentences and label conditions are really simple compared with composition rules of toy sentences and toy conditions. The followings are the composition rules of label sentences and label conditions in basic toy language.

5.3.1. Any expression of the form $x \cdots y \mathrm{P}$ for any primitive notion $\mathrm{P}$ is a label sentence.

5.3.2. Any expression of the form $\forall x \cdots y \frac{F, \cdots, G}{H}$ for any non-repetitive series $x$, $\cdots, y$, any label sentence series $F, \cdots, G$, and any label sentence $H$ is a label sentence.

5.3.3. Any expression of the form $[x, \cdots, y / F]$ for any non-repetitive series $x, \cdots, y$ of the length $\mathrm{k}$ and any label sentence $\mathrm{F}$ is a label condition on $\mathrm{k}$ variables, occasionally represented in the form $[\mathrm{k} ! \mathrm{C}]$.

Any expression of the form $u \cdots v[\mathrm{k} ! C]$ for any series $u, \cdots, v$ of the length $\mathrm{k}$ is $a$ label sentence. $(\mathrm{k}=0,1, \cdots)$

5.4. By the following facts (5.4.1-5.4.4), we can use label sentences and label conditions to represent toy propositions and toy notions, respectively. 
5.4.1. For any toy proposition, we can find out its label being a label sentence.

5.4.2. For any $\mathrm{k}$ variable toy notion, we can find out its label being a label condition on $\mathrm{k}$ variables. $(\mathrm{k}=0,1, \cdots)$

5.4.3. For any label sentence, we can find out a toy proposition having the label sentence as its label.

5.4.4. For any $\mathrm{k}$ variable label condition, we can find out $a \mathrm{k}$ variable toy notion having the label condition as its label.

5.5. Hereafter, we deal with deducibility of toy propositions rather than that of toy sentences. We represent toy boxes occasionally by their labels and occasionally by their samples.

5.5.1. We call any pair of label sentences (or label conditions) similar if and only if they are labels of the same toy box.

5.5.2. Let $\Omega$ be any finite list of letters for object variables. Then,

1) Any label sentence $A$ of the form $\forall x \cdots y \frac{F, \cdots, G}{H}$ has a label sentence of the form $\forall u \cdots v \frac{F^{*}, \cdots, G^{*}}{H^{*}}$ similar to $A$ such that $u, \cdots, v$ do not belong to $\Omega$.

2) Any label condition $[\mathrm{k} ! C]$ of the form $[x, \cdots, y / F]$ has a label condition of the form $\left[u, \cdots, v / F^{*}\right]$ similar to $[\mathrm{k} ! C]$ such that $u, \cdots, v$ do not belong to $\Omega$.

5.5.3. To be practical, labels may be expressed in other forms assuming that we are confident of reproducing the original forms. For example,

1) We may omit some parentheses in labels if no fear of ambiguity is expected.

2) Label sentences of the form $\forall x \cdots y-\frac{}{C}$ are usually expressed in the form $\forall x \cdots y(C)$.

3) Label sentences of the form $\forall x \cdots y \frac{F, \cdots, G}{H}$ may be occasionally express-

ed in the form $\forall x \cdots y \frac{\left(\begin{array}{l}F, \cdots, \\ \cdots, \cdot) \\ \cdots, G\end{array}\right)}{H}$.

4) Label sentences of the form $\forall \frac{F, \cdots, G}{H}$ and toy propositions of the form $\forall \frac{\mathbf{F}, \cdots, \mathbf{G}}{\mathbf{H}}$ may be occasionally expressed in the forms $(F, \cdots, G) \rightarrow H$ and $(\mathbf{F}, \ldots, \mathbf{G}) \rightarrow \mathbf{H}$. respectively.

5.5.4. Occasionally, we call some toy notions by their nicknames, or we denote them by their nickname symbols. Occasionally, we express labels in terms of nickname symbols. 


\section{Toy boxes in the world of objects coming under a toy notion}

For any single variable notion and any proposition in our ordinary language, we are apt to think of the proposition in the world of objects coming under the notion. For example, we are apt to think of a proposition in the world of figures in a space, etc.

6.1. In any basic toy language, let $[1 ! \mathrm{N}]$ be any toy notion with a label $[1 ! N]$, and let $\mathbf{B}$ be any toy box with a label $B$. Then, we denote by $\mathbf{B}^{[1 / N]}$ the toy box in the world of objects coming under the toy notion [1!N].

We use symbols of the form $B^{[11 N]}$ for communication to show forms of labels of the toy box $\mathbf{B}^{[11 \mathrm{~N}]}$.

Let $\Omega$ be any finite list of letters containing all the object variables occurring free in $[1 ! N]$. Then, we can introduce the toy box $\mathbf{B}^{[1 ! N]}$ for any toy box $\mathbf{B}$ as follows: (6.1.1-6.1.4.)

6.1.1. Let $\mathrm{B}$ be any toy proposition with a label of the form $x \cdots y \mathrm{P}$ for a primitive notion $\mathbf{P}$. Then, $\mathbf{B}^{[11 N]}$ is the toy proposition $\mathbf{B}$ itself.

6.1.2. Let $\mathbf{B}$ be any toy proposition with a label of the form $\forall x \cdots y \frac{F, \cdots, G}{H}$ suh that $x, \cdots, y$ do not belong to $\Omega$. (cf. 5.5.2.) Then, $\mathbf{B}^{[11 N]}$ is the toy proposition with labels of the form $\forall x \cdots y \frac{x[1 ! N], \cdots, y[1 ! N], F^{[11 N]}, \cdots, G^{[1 \mid N]}}{H^{[1 \mid N]}}$

6.1.3. Let $\mathbf{B}$ be any toy notion with a label of the form $\left[x, \cdots, y \mid F^{\prime}\right]$ such that $x, \cdots, y$ do not belong to $\Omega$. (cf. 5.5.2.) Then, $\mathbf{B}^{[11 N]}$ is the toy notion with labels of the form $\left[x, \cdots, y / F^{[11 N]}\right]$.

6.1.4. Let $\mathbf{B}$ be a toy proposition with a label of the form $u \cdots v[\mathbf{k} ! C]$. Then, $\mathbf{B}^{[1+N]}$ is the toy proposition with labels of the form $u \cdots v\left([\mathrm{k} ! C]^{[11 N]}\right)$.

\section{Toward handy toy languages}

In our ordinary language, we use negation, conjunction, disjunction, existential quantification, etc. together with general implication as composition tools.

In any basic toy language, there are some partial languages, in which these composition tools can be defined with respect to each toy notion so that most of logical rules are preserved. By choosing toy notions suitably, we can see that all the logical rules can be preserved.

It would be a matter of course to think of a toy language having these composition tools and logical rules common to all the partial languages defined with respect to a certain class of toy notions.

Thus, we are led to imagine a series of toy languages, which we call intuitinoistic, classical, and handy toy languages in this paper.

Introduction of logical constants is along the way of [3] and [4]. 


\section{1. $[k$ ! Q]-languages}

In any basic toy language, let us take up any toy notion [k! Q] with a label $[k ! Q] .(k=0,1, \cdots)$

7.1.1. We denote by $F_{[\mathrm{k} \mid \mathrm{Q}]}$ for any toy proposition $\mathbf{F}$, the toy proposition $\forall x \cdots y \frac{\mathbf{F} \rightarrow x \cdots y[\mathbf{k} ! \mathbf{Q}]}{x \cdots y[\mathrm{k} ! \mathbf{Q}]}$ for any non-repetitive series $x, \cdots, y$ of the length $k$ such that $x, \cdots, y$ do not occur in $[\mathrm{k} ! \mathrm{Q}]$.

7.1.2. We call any toy proposition $F$ to be [k!Q]-invariant if and only if $F$ is deducible from $\mathrm{F}_{[\mathrm{k} \mid \mathrm{Q}]}$.

We call any toy notion of the form $[x, \cdots, y / \mathbf{F}]$ to be $[\mathrm{k} ! \mathbf{Q}]$-invariant if and only if $\mathbf{F}$ is a $[\mathbf{k} ! \mathbf{Q}]$-invariant toy proposition.

7.1.3. We can prove that $\mathbf{F}_{[\mathrm{k} ! \mathbf{Q}]}$ is [k!Q]-invariant for any toy propositin $\mathbf{F}$.

7.1.4. We can prove that any toy proposition $\forall x \cdots y \frac{\mathbf{F}, \cdots, \mathbf{G}}{\mathbf{H}}$ for any $[k ! \mathbf{Q}]$-invariant toy proposition $\mathbf{H}$ and any non-repetitive series $x, \cdots, y$ having no term occurring free in labels $\boldsymbol{F}, \cdots, \boldsymbol{G}, \boldsymbol{H}$ of $\mathbf{F}, \cdots, \mathbf{G}, \mathbf{H}$, respectively, is [k! Q]-invariant.

7.1.5. We introduce [k! $\mathbf{Q}]$-propositions as follows:

1) For any elementary toy proposition $\mathbf{E}, \boldsymbol{E}_{[\mathbf{k} 1 \mathbf{Q}]}$ is a [k!Q]-proposition.

2) Any toy proposition of the form $\forall x \cdots y \frac{\mathbf{F}, \cdots, \mathbf{G}}{\mathbf{H}}$ for any $[\mathbf{k} ! \mathbf{Q}]-$ propositions $\mathbf{F}, \cdots, \mathbf{G}, \mathbf{H}$ and any non-repetitive series $x, \cdots, y$ having to term occurring free in $[\mathrm{k} ! \mathbf{Q}]$ is a $[\mathbf{k} ! \mathbf{Q}]$-proposition.

7.1.6. We call any toy notion $\mathbf{N}$ a $[\mathrm{k}$ ! Q]-notion if and only if $\mathbf{N}$ is a toy notion of the form $[x, \cdots, y / \mathbf{F}]$ for any $[\mathbf{k} ! \mathbf{Q}]$-proposition $\mathbf{F}$ and any non-repetitive series $x, \cdots, y$ having no term occuring free in $[\mathrm{k} ! \mathrm{Q}]$.

7.1.7. The partial language formed by all the [k!Q]-propositions and all the [k!Q]notions is called a [ $\mathrm{k} ! \mathrm{Q}]$-language.

7.1.8. We can prove that any [k!Q]-proposition is deducible from itself.

7.2. Composition tools in [k!Q]-languages

In any [k! Q]-language, we can introduce negation $\rangle_{[\mathbf{k} \mid \mathbf{Q}]}$, conjunction $\wedge_{[\mathbf{k} \mid \mathbf{Q}]}$, disjunction $V_{[\mathbf{k} \mid \mathbf{Q}]}$, and existential quantification $\exists_{[\mathbf{k} \mid \mathbf{Q}]}$ with respect to $[\mathrm{k} ! \mathbf{Q}]$.

7.2.1. $>_{[\mathrm{k} \mid \mathrm{Q}]}(\mathbf{F})$ is the toy proposition $\forall x \cdots y \frac{\mathbf{F}}{x \cdots y[\mathrm{k} ! \mathbf{Q}]}$ for any non-repetitive series $x, \cdots, y$ having no term occurring free in labels $[\mathrm{k} ! Q]$ and $F$ of $[\mathrm{k} ! \mathrm{Q}]$ and F, respectively.

$7_{[\mathbf{k} \mathbf{Q}]}(\mathbf{F})$ for any [k!Q]-proposition $F$ is surely a [k!Q]-proposition.

We express the form of labels of $\rangle_{[\mathbf{k} \mid Q]}(\mathbf{F})$ by $\rangle_{[\mathbf{k} \mid Q]}(F)$.

7.2.2. $\wedge \wedge_{[\mathbf{k} \mid \mathbf{Q}]}(\mathbf{F}, \cdots, \mathbf{G})$ is the toy proposition $\forall x \cdots y \frac{(\mathbf{F}, \cdots, \mathbf{G}) \rightarrow x \cdots y[\mathrm{k} ! \mathbf{Q}]}{x \cdots y[\mathbf{k} ! \mathbf{Q}]}$ for any non-repetitive series $x, \cdots, y$ having no term occurring free in labels $[k ! Q]$, 
$F, \cdots, G$ of $[\mathrm{k} ! \mathrm{Q}], \mathrm{F}, \cdots, \mathrm{G}$, respectively.

$\wedge[k \mid Q](F, \cdots, G)$ for any $[k ! Q]$-propositions $F, \cdots, G$ is surely a $[k ! Q]$-proposition.

We express the form of labels of $\wedge_{[\mathrm{k} \mid \mathrm{q}]}(\mathrm{F}, \cdots, \mathrm{G})$ by $\wedge_{[\mathrm{k} \mid \mathrm{Q}]}(F, \cdots, G)$.

7.2.3. $\vee_{[\mathbf{k} \mid \mathbf{Q}]}(\mathbf{F}, \cdots, \mathbf{G})$ is the toy proposition $\forall x \cdots y \frac{F \rightarrow x \cdots y[\mathrm{k} ! \mathbf{Q}], \cdots, G \rightarrow x \cdots y[\mathrm{k} ! \mathbf{Q}]}{x \cdots y[\mathbf{k} ! \mathbf{Q}]}$ for any non-repetitive series $x, \cdots, y$ having no term occurring free in labels $[\mathrm{k} ! Q]$, $F, \cdots, G$ of $[\mathrm{k} ! \mathbf{Q}], \mathbf{F}, \cdots, \mathrm{G}$, respectively.

$V_{[k ! Q]}(\mathbf{F}, \ldots, G)$ for any $[k ! \mathbf{Q}]$-propositions $\mathbf{F}, \ldots, \mathbf{G}$ is surely a $[\mathrm{k} ! \mathbf{Q}]$-proposition.

We express the form of labels of $\vee_{[k \mid Q]}(\mathbf{F}, \ldots, G)$ by $\vee_{[k] Q]}(F, \cdots, G)$.

7.2.4. $\exists_{[k \mid Q]} s \cdots t(\mathbf{F}, \cdots, G)$ for any non-repetitive series $s, \cdots, t$ having no term occurring free in $[\mathrm{k} ! \mathbf{Q}]$ is the toy proposition $\forall x \cdots y \frac{\forall s \cdots t \frac{\mathbf{F}, \cdots, \mathbf{G}}{x \cdots y[\mathrm{k} ! \mathbf{Q}]}}{x \cdots y[\mathbf{k} ! \mathbf{Q}]}$ for any non-repetitive series $x, \cdots, y$ having no term occurring free in labels $[\mathrm{k} ! Q], F, \cdots, G$ of $[k ! \mathbf{Q}], \mathbf{F}, \cdots, \mathbf{G}$, respectively.

$\exists_{[k ! Q]} s \cdots t(\mathbf{F}, \cdots, G)$ for any $[k ! Q]$-propositions $F, \cdots, G$ is surely a $[k ! Q]-$ proposition.

We express the form of labels of $\exists_{[k \mid a]^{s}} \cdots t(\mathbf{F}, \cdots, \mathbf{G})$ by $\exists_{[k \mid Q}[]^{s} \cdots t(F, \cdots, G)$.

7.3. In any [k! Q]-language, we can prove the followings: (7.3.1-7.3.8.)

7.3.1. $7_{[\mathbf{k} 1 \mathbf{Q}]}(\mathbf{F})$ is deducible from $\mathbf{F} \rightarrow \mathbf{G}$ and $\mathbf{F} \rightarrow>_{[\mathbf{k} 1 \mathbf{Q}]}(\mathbf{G})$.

7.3.2. $\mathbf{F}$ is deducible from $\mathbf{G}$ and $\rangle_{[\mathbf{k} \mid \mathbf{Q}]}(\mathbf{G})$.

7.3.3. $\wedge \wedge_{[\mathbf{k}(\mathbf{Q}]}(\mathbf{F}, \cdots, \mathbf{G})$ is deducible from $\mathbf{F}, \cdots, \mathbf{G}$.

7.3.4. $\mathbf{F}$ is deducible from $\wedge_{[\mathrm{k} \mid \mathrm{Q}]}(\mathbf{F}, \cdots, \mathbf{G})$.

7.3.5. $V_{[\mathbf{k} 1 \mathbf{Q}]}(\cdots, \mathbf{F}, \cdots)$ is deducible from $\mathbf{F}$.

7.3.6. $\mathbf{H}$ is deducible from $\mathbf{F} \rightarrow \mathbf{H}, \cdots, \mathbf{G} \rightarrow \mathbf{H}$, and $\vee_{[\mathrm{k} \mid \mathbf{Q}]}(\mathbf{F}, \cdots, \mathbf{G})$.

7.3.7. $\Xi_{[\mathbf{k} \mid \mathbf{Q}]} x \cdots y(\mathbf{F}, \cdots, \mathbf{G})$ is deducible from $[\mathrm{k} ! \mathbf{Q}]$-propositions represented by samples of the forms $\left(\begin{array}{l}\boldsymbol{x}, \cdots, \boldsymbol{y} \\ \mathbf{u}, \cdots, \mathbf{v}\end{array}\right)(\mathscr{F}), \cdots,\left(\begin{array}{l}\boldsymbol{x}, \cdots, \boldsymbol{y} \\ \mathbf{u}, \cdots, \mathbf{v}\end{array}\right)(\mathscr{G})$ for any samples $\mathscr{F}, \cdots, \mathscr{G}$ of the $[\mathrm{k} ! \mathbf{Q}]$-propositions $\mathbf{F}, \cdots, \mathbf{G}$, respectively.

7.3.8. Any $[\mathrm{k} ! \mathbf{Q}]$-proposition $\mathbf{H}$ with labels having no free occurrence of $x, \cdots, y$ is deducible form $\forall x \cdots y \frac{\mathbf{F}, \cdots, \mathbf{G}}{\mathbf{H}}$, and $\Xi_{[\mathbf{k}: \mathbf{q}]} x \cdots y(\mathbf{F}, \cdots, \mathbf{G})$.

7.4. For any toy notion of the form [0! Q], we can prove that any [0!Q]-proposition $\mathbf{F}$ is deducible from $7_{[010]}\left(7_{[010]}(\mathbf{F})\right)$.

7.5. To deal with toy propositions in the world of objects coming under a single variable toy notion agreeably, we are led to deal with [0! Q]-propositions for a more restricted kind of toy notions, i.e. quantification-free toy notions [0! $\mathbf{Q}]$. 
Quantification-free toy propositions and toy notions in basic toy language can be introduced as follows:

(1) Any elementary toy proposition is quantification-free.

(2) Any toy proposition of the form $(\mathbf{F}, \ldots, \mathbf{G}) \rightarrow \mathbf{H}$ for any quantificationfree toy propositions $\mathbf{F}, \ldots, \mathbf{G}, \mathbf{H}$ is quantification free.

(3) Any toy notion of the form $[x, \cdots, y / \mathbf{F}]$ for any quantification-free toy proposition $\mathbf{F}$ is quantification-free.

(4) Any toy proposition of the form $x \cdots y[\mathrm{k} ! \mathrm{C}]$ for any quantification-free toy notion $[\mathrm{k} ! \mathrm{C}]$ is quantification-free.

Now, let $[0 ! \mathbf{Q}]$ be quantification-free, and $[1 ! \mathbf{N}]$ be any $[0 ! \mathbf{Q}]$-notion. Then, in any $[0 ! Q]$-language, we can prove the following: (7.5.1-7.5.4.)

7.5.1. $\left(7_{[01 Q]}(\mathbf{F})\right)^{[1 / N]}$ and $\rangle_{[01 Q]}\left(F^{[1 ! N]}\right)$ are deducible from each other.

7.5.2. $\left.\wedge_{[0 \mid Q]}(\mathbf{F}, \ldots, \mathbf{G})\right)^{[11 \mathbb{N}]}$ and $\wedge_{[0 \mid Q]}\left(\mathbf{F}^{[11 \mathbb{N}]}, \ldots, \mathbf{G}^{[1 \mid \mathbb{N}]}\right)$ are deducible from each other.

7.5.3. $\left(V_{[0 \mid Q]}(F, \cdots, G)\right)^{[11 \mathbb{N}]}$ and $V_{[01 Q]}\left(F^{[1 \mid N]}, \cdots, G^{[1 / N]}\right)$ are deducible from each other.

7.5.4. For any non-repetitive series $x, \cdots, y$ having no term occurring free in labels of $[1-\mathbf{N}],\left(\exists_{[0 \mid Q]} x \cdots y(\mathbf{F}, \cdots, \mathbf{G})\right)^{[1 ! N]}$ and $\exists_{[0 \mid Q]} x \cdots y\left(x[1 ! \mathbf{N}], \cdots, y[1 ! \mathbf{N}], F^{[1 \mid N]}\right.$, $\cdots, G^{[1 / N]}$ ) are deducible from each other.

\subsection{Imagination of intuitionistic toy language}

According to 7.1.8 and 7.3.1-7.3.8, we can see that [k!Q]-languages behave in parallel with each other satisfying inference rules of the usual intuitionistic logic. Presumptuous or not, we dare to imagine a toy language behind all these [k!Q]languages behaving in parallel with each other. We will call the imagined toy language intuitionistic toy language in this paper.

Intuitionistic toy language can be introduced as follows: (7.6.1.-7.6.2.)

7.6.1. Toy sentences and toy conditions are introduced as follows:

(1) Any expression of the form $x \cdots y \mathrm{P}$ for any primitive notion $\mathrm{P}$ is a toy sentence.

(2) Any expression of any one of the forms $\forall \mathbf{x} \cdots \mathbf{y}\left(\left(\begin{array}{l}x, \cdots, y \\ \mathbf{x}, \cdots, y\end{array}\right)\left(\frac{\mathscr{F}, \cdots, \mathscr{G}}{\mathcal{H}}\right)\right)$ and $\exists \mathbf{x} \cdots \mathbf{y}\left(\left(\begin{array}{c}x, \cdots, y \\ \mathbf{x}, \cdots, y\end{array}\right)(\mathscr{F}, \cdots, \mathscr{G})\right)$ for any toy sentences $\mathscr{F}, \cdots, \mathscr{G}, \mathcal{H}$, any nonrepetitive series $\mathbf{x}, \cdots, \mathbf{y}$ having no term occurring in $\mathscr{F}, \cdots, \mathscr{G}, \mathcal{H}$, and any nonrepetitive series $x, \cdots, y$ of the same length as $\mathbf{x}, \cdots, \mathbf{y}$ is a toy sentence.

(3) Any expression of any one of the forms $>(\mathscr{F}), \wedge(\mathscr{F}, \ldots, \mathscr{G})$, and $\mathrm{V}(\mathscr{F}, \ldots, \mathscr{E})$ for any sentences $\mathscr{F}, \ldots, \mathscr{G}$ is a toy sentence.

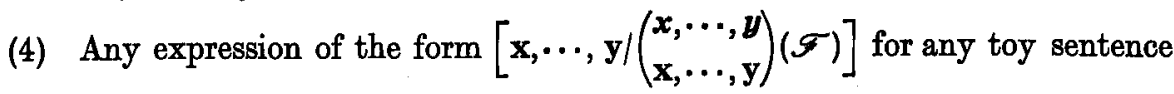
$F$, any non-repetitive series $\mathbf{x}, \ldots, \mathbf{y}$ of the length $\mathbf{k}$ having no term occurring in $\mathscr{F}$, 
and any no-repetitive series $x, \cdots, y$ of the length $\mathbf{k}$ is a $\mathbf{k}$ variable toy condition.

We use notations of the form $[k ! \mathscr{E}]$ for $k$ variable toy conditions. $(1=0,1 \ldots)$

(5) Any expression of the form $u \cdots v[k ! \mathscr{E}]$ for any series $u, \cdots, v$ of the length $\mathrm{k}$ is a toy sentence. $(\mathrm{k}=0,1, \cdots)$

(6) Any expression of the form $u \cdots v[k ! \mathscr{E}]$ for any series $u, \cdots, v$ of the

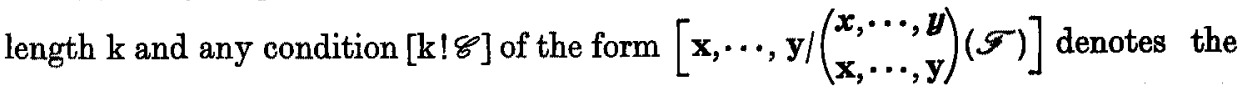
toy sentence $\left(\begin{array}{l}\boldsymbol{x}, \cdots, \boldsymbol{v} \\ \boldsymbol{u}, \cdots, \boldsymbol{v}\end{array}\right)(\mathscr{F}) . \quad(\mathrm{k}=0,1, \cdots)$

7.6.2. In intuitionistic toy language, we have the following inference rules:

(1) Any toy sentence is deducible from itself. (cf. 7.1.8.)

(2) Any toy sentence of the form $\forall \mathbf{x} \cdots \mathbf{y}\left(\left(\begin{array}{l}\mathbf{x}, \cdots, \boldsymbol{y} \\ \mathbf{x}, \cdots, \mathbf{y}\end{array}\right)\left(\frac{\mathscr{F}, \cdots, \mathscr{E}}{\mathcal{H}}\right)\right)$ for any toy sentences $\mathscr{F}, \cdots, \mathscr{G}, \mathcal{H}$ is deducible if $\left(\begin{array}{l}\boldsymbol{x}, \cdots, \boldsymbol{y} \\ \boldsymbol{u}, \cdots, \boldsymbol{v}\end{array}\right)(\mathcal{H})$ is deducible from $\left(\begin{array}{l}\boldsymbol{x}, \cdots, \boldsymbol{y} \\ \boldsymbol{u}, \cdots, \boldsymbol{v}\end{array}\right)(\mathscr{F}) \cdots$, $\left(\begin{array}{l}x, \cdots, \boldsymbol{y} \\ u, \cdots, v\end{array}\right)(\mathscr{G})$ for any arbitrary $u, \cdots, v$ of the same length as $x, \cdots, u$. (cf. 4.4.2.)

(3) From any series of toy sentences of the forms $\forall x, \cdots y\left(\left(\begin{array}{l}x, \cdots, y \\ \mathbf{x}, \cdots, \mathbf{y}\end{array}\right)\left(\frac{\mathscr{F}, \cdots, \mathscr{G}}{\mathcal{H}}\right)\right)$, $\left(\begin{array}{l}\boldsymbol{x}, \cdots, \boldsymbol{y} \\ \boldsymbol{u}, \cdots, \boldsymbol{v}\end{array}\right)(\mathscr{F}), \cdots,\left(\begin{array}{l}\boldsymbol{x}, \cdots, \boldsymbol{v} \\ \boldsymbol{u}, \cdots, \boldsymbol{v}\end{array}\right)(\mathscr{E})$ for any series $\boldsymbol{u}, \cdots, \boldsymbol{v}$ of the same length as $\boldsymbol{x}, \cdots, \boldsymbol{y}$, the toy sentence $\left(\begin{array}{l}\boldsymbol{x}, \cdots, \boldsymbol{y} \\ \boldsymbol{u}, \cdots, \boldsymbol{v}\end{array}\right)(\mathscr{H})$ is deducible. (cf. 4.4.3.)

(4) $>\mathscr{F}$ is deducible from $\mathscr{F} \rightarrow \mathscr{G}$ and $\mathscr{F} \rightarrow(\neg \mathscr{G})$ for any toy sentences $\mathscr{F}$ and $\mathscr{G}$. (cf. 7.3.1.)

(5) $\mathscr{F}$ is deducible from $\mathscr{G}$ and $>\mathscr{G}$ for any toy sentences $\mathscr{F}$ and $\mathscr{G}$. (cf. 7.3.2.)

(6) $\wedge(\mathscr{F}, \ldots, \mathscr{G})$ is deducible from $\mathscr{F}, \ldots, \mathscr{G}$ for any toy sentences $\mathscr{F}, \ldots, \mathscr{G}$. (cf.

(7) $\mathscr{F}$ is deducible from $\wedge(\cdots, \mathscr{F}, \cdots)$ for any toy sentences $\cdots, \mathscr{F}, \cdots$ (cf. 7.3.4.)

(8) $\vee(\cdots, \mathscr{F}, \cdots)$ is deducible from $\mathscr{F}$ for any toy sentences $\cdots, \mathscr{F}, \cdots$ (cf. 7.3.5.)

(9) $\mathcal{X}$ is deducible from $\mathscr{F} \rightarrow \mathcal{H}, \cdots, \mathscr{G} \rightarrow \mathcal{X}$ and $\vee(\mathscr{F}, \cdots, \mathscr{G})$ for any toy sentences $\mathscr{F}, \cdots, \mathscr{G}, \mathcal{H}$. (cf. 7.3.6.)

(10) Any toy sentence of the form $\exists \mathbf{x} \cdots \mathbf{y}\left(\left(\begin{array}{l}x, \cdots, y \\ \mathbf{x}, \cdots, y\end{array}\right)(\mathscr{F}, \cdots, \mathscr{G})\right)$ is deducible from $\left(\begin{array}{l}\boldsymbol{x}, \cdots, \boldsymbol{v} \\ \boldsymbol{u}, \cdots, \boldsymbol{v}\end{array}\right)(\mathscr{F}), \cdots,\left(\begin{array}{l}\boldsymbol{x}, \cdots, \boldsymbol{y} \\ \boldsymbol{u}, \cdots, \boldsymbol{v}\end{array}\right)(\mathscr{E})$ for any toy sentences $\mathscr{F}, \cdots, \mathscr{S}$, any non-repetitive series $\boldsymbol{x}, \cdots, \boldsymbol{y}$, and any series $\boldsymbol{u}, \cdots, \boldsymbol{v}$ of the same length as $\boldsymbol{x}, \cdots, \boldsymbol{y}$. (cf. 7.3.7.)

(11) $\mathcal{H}$ is deducible from any toy sentences of the forms $\forall \mathrm{x} \cdots \mathrm{y}\left(\left(\begin{array}{l}x, \cdots, y \\ \mathbf{x}, \cdots, y\end{array}\right)(\mathscr{F}, \cdots, \mathscr{G} \rightarrow \mathscr{H})\right)$ and $\mathrm{gx} \cdots \mathrm{y}\left(\left(\begin{array}{l}x, \cdots, \boldsymbol{y} \\ \mathbf{x}, \cdots, \mathrm{y}\end{array}\right)(\mathscr{F}, \cdots, \mathscr{E})\right)$ for any sen- 
tences $\mathscr{F}, \cdots, \mathscr{G}, \mathcal{K}$, and any non-repetitive series $\mathbf{x}, \cdots, \mathbf{y}$ having no term occurring in H. (cf. 7.3.8.)

\section{7. 'Imagination of classical toy language}

According to 7.1.8, 7.3.1-7.3.8, and 7.4, we can see the [0!Q]-languages behave in parallel with each other satisfying inference rules of the usual classical logic. Presumptuous or not, we dare to imagine a toy language behind all the [0!Q]langauges behaving in parallel with each other. We will call the imagined toy language classical toy language in this paper.

Classical toy language is introduced as follows:

7.7.1. Toy sentences and toy conditions of classical toy language are the same as that of intuitionistic toy language. (See 7.6.1.)

7.7.2. In classical toy language, we can prove the following inference rule:

(12) $\mathscr{F}$ is deducible from $>(>\mathscr{F}$ ) for any toy sentence $\mathscr{F}$. (cf. 7.4.)

together with all the inference rules of intuitionistic toy language. (See 7.6.2, (1)-(11).)

\subsection{Imagination of handy toy language}

According to 7.1.8, 7.3.1-7.3.8,7.4, 7.5, and 7.5.1-7.4, we can see that [0! Q]-languages for any quantification-free toy notion [0! Q] behave in parallel with each other satisfying all the inference rules of the usual classical logic and composition rules of propositions in the world of objects coming under a single variable toy notion.

Presumptuous or not, we dare to imagine a toy language behind all the [0!Q] -languages for quantification-free [0!Q]'s behaving in parallel with each other. We will call the imagined toy language handy toy language in this paper.

Handy toy language is introduced as follows:

7.8.1. All the toy sentences and toy conditions of intuitionistic toy language introduced by (1) $-(6)$ of 7.6.1. are toy sentences and toy conditions of handy toy language. Other than these, we admit the following composition rules:

(7) For any toy sentence $\mathscr{F}$ and any toy condition $[1 ! \mathscr{N}]$, the expression $\left.F^{[11} \boldsymbol{r}\right]$ is a toy sentence.

(8) $[k ! \mathscr{E}]^{[1 ! \mathscr{R}]}$ for any toy conditions $[\mathrm{k} ! \mathscr{C}]$ and $[1 ! \mathscr{N}]$ is a $\mathrm{k}$ variable toy condition. $(k=0,1, \cdots)$

8.8.2. In handy toy language, we can prove the following inference rules:

(13) For any toy sentence $F$ of the form $x \cdots y \mathrm{P}$ for any primitive notion $\mathrm{P}$, $\mathscr{F}^{[11 \mathcal{R}]}$ and $\mathscr{F}$ are mutually deducible for any $[1 ! \mathfrak{S}]$.

(14) Toy sentences of the forms $\left(\forall \mathbf{u} \cdots \mathbf{v}\left(\left(\begin{array}{l}x, \cdots, \boldsymbol{y} \\ \mathbf{u}, \cdots, \mathbf{v}\end{array}\right)\left(\frac{\mathscr{F}, \cdots, \mathscr{G}}{\mathscr{H}}\right)\right)\right)^{[11 \boldsymbol{r}]}$ and $\forall \mathbf{s} \cdots \mathrm{t}\left(\left(\begin{array}{l}x, \cdots, y \\ \mathrm{~s}, \cdots, \mathrm{t}\end{array}\right)\left(\frac{\mathrm{s}[1 ! \mathscr{r}], \cdots, \mathrm{t}[1 ! \mathscr{N}], \mathscr{F}[11 \cdot r], \cdots, \mathscr{G}^{[11 \cdot r]}}{\mathscr{H}^{[1 ! \mathscr{r}]}}\right)\right)$ are deducible from each 
other for any toy sentences $\mathscr{F}, \ldots, \mathscr{G}, \mathcal{H}$, any toy condition of the form [1! $\mathscr{N}]$, any non-repetitive series $\mathbf{x}, \cdots, \mathbf{y}$ having no term occurring in $[1 ! \mathscr{N}]$, and any series $\mathbf{u}, \cdots, \mathbf{v}$ and $\mathbf{s}, \cdots, \mathrm{t}$ of the same lengths as $\boldsymbol{x}, \cdots, \boldsymbol{y}$ and having no term occurring in $\mathscr{F}, \cdots, \mathscr{E}$, $\mathcal{H},[1 ! \mathscr{N}]$. (of. 6.1.2. and 7.1.8.)

(15) $\left(\left(\mathcal{F}^{\mathscr{F}}\right)\right)^{[11 \boldsymbol{r}]}$ and $>\left(\mathscr{F}^{\left[11 \boldsymbol{r}^{]}\right]}\right.$) are deducible from each other for any toy sentence $\mathscr{F}$ and any toy condition of the form $[1 ! \mathscr{N}]$. (cf. 7.5.1.)

(16) $(\wedge(\mathscr{F}, \cdots, \mathscr{G}))$ and $\wedge\left(\mathscr{F}^{[\mathrm{u} \sim \boldsymbol{}]}, \ldots, \mathscr{G}^{[11 \cdot r]}\right)$ are deducible from each other for any toy sentences $\mathscr{F}, \cdots, \mathscr{G}$, and any toy condition of the form [1! $\mathscr{N}]$. (cf. 7.5.2.)

(17) $(\vee(\mathscr{F}, \ldots, \mathscr{G}))^{[11 \sim r}$ and $\vee\left(\mathscr{F}^{[11 \mathscr{r}]}, \ldots, \mathscr{S}^{[11 \mathcal{N}]}\right)$ are deducible from each other for any toy sentences $\mathscr{F}, \ldots, \mathscr{G}$, and any toy condition of the form [1! N]. (cf. 7.5.3.)

(18) Any toy sentence of the form $\left(\exists \mathrm{u} \cdots \mathrm{v}\left(\left(\begin{array}{c}x, \cdots, \boldsymbol{v} \\ , \mathrm{u} \cdots, \mathrm{v}\end{array}\right)(\mathscr{F}, \cdots, \mathscr{G})\right)\right)^{[11 \mathscr{r}]}$ and any

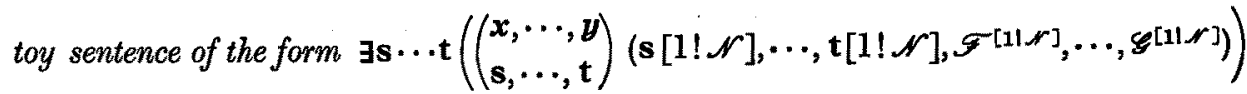
are deducible from each other for any toy sentences $\mathscr{F}, \cdots, \mathscr{G}$, any toy condition of the form $[1 ! \mathscr{N}]$, any non-repetitive series $\mathbf{u}, \cdots, \mathbf{v}$ and $\mathbf{s}, \cdots, \mathbf{t}$ of the same lengths as $\boldsymbol{x}, \cdots$, $\boldsymbol{y}$ and having no term occurring in $\mathscr{F}, \cdots, \mathscr{S},[1-\mathscr{N}]$. (cf. 7.5.4.)

(19) For any toy sentence of the form $x \cdots y[\mathrm{k} ! \mathscr{E}]$ and any toy condition of the form $[1 ! \mathscr{N}]$, the toy sentences $(\boldsymbol{x} \cdots \boldsymbol{y}[\mathrm{k} ! \mathscr{E}])^{[1 ! \cdot \boldsymbol{r}]}$ and $\boldsymbol{x} \cdots \boldsymbol{y}\left([\mathrm{k} ! \mathscr{E}]^{[1 ! \cdot \boldsymbol{r}]}\right)$ are deducible from each other. (cf. 6.1.3, 6.1.4, and 7.1.8.)

together with all the inference rules of classical toy language. (See (1)-(11) of 7.6.2. and (12) of 7.7.2.)

7.9. Toy propositions, toy notions, and their labels in intuitionistic, classical, or handy toy languages.

In intuitionistic, classical, or handy toy languages, we can introduce similarity concept just as in basic toy language, and we can prove that similar toy sentences as well as similar toy conditions are logically equivalent to each other in respective languages. Accordingly, we can introduce toy propositions, toy notions, and their labels in these toy languages just as we have done in basic toy language.

In inutitionstic, clasical, or handy toy language, we are also led to the mood of dealing with toy propositions and toy notions, occasionally by their labels, occasionally by their samples.

7.9.1. In intuitionistic, classical, or handy toy languages, it is occasionally practical to introduce equivalence $\equiv$ as follows:

We denote by $F \equiv G$ the label sentence form $\wedge(F \rightarrow G, G \rightarrow F)$ for any label sentences $F$ and $G$.

7.9.2. In intuitionistic, classical, or handy toy languages, it is occasionally practical to express labels of the forms $\wedge(F, \cdots, G), \vee(F, \cdots, G)$, and $\exists x \cdots y(F, \cdots, G)$ in the 
forms $\wedge\left(\begin{array}{c}F, \cdots \\ \cdots \\ \cdots, G\end{array}\right), \vee\left(\begin{array}{c}F, \cdots \\ \cdots \\ \cdots, G\end{array}\right)$, and $\exists x \cdots y\left(\begin{array}{c}F, \cdots \\ \cdots \\ \cdots, G\end{array}\right)$, respectively.

\section{Imaginative toy language}

Real reasonings in mathematics seem to be nicely copied in handy toy language with some toy principles which are also faithful copies of tacitly assumed principles of our real resonings.

In mathematics, we are apt to think of sets. Any set is nothing but an object representing a single variable condition. More in general, we are apt to think of objects representing conditions on any number of variables.

In our real thinking, we are apt to think of propositions in the form

" $x, \cdots, y$ satisfy the condition $C, "$

and further we try to represent the condition $C$ by an object $z$. When we are successful to do so, the proposition turns out to be

" $x, \cdots, y$ satisfy the condition represented by $z . "$

Regarding this in the form $x \cdots y z \mathrm{~B}$, we are led to imagine a basic primitive notion $B$, which seems to be a copy of the verb "be".

In our real thinking, we are also tacitly assuming equality notion $\mathrm{E}$ such that $x y \mathrm{E}$ means $x=y$ in usual notations.

Here, we would like to exhibit a trial toy language called imaginative language in this paper having a few toy principles with respect to $B$ and $E$, which seem to be copies of principles tacitly assumed in our real thinking. We are expecting that our real mathematical thinking can be nicely described in our imaginative toy language.

8.1. Labels $[p]_{\mathrm{k}}, \subset_{\mathrm{k}}, \in_{\mathrm{k}},\left[\in_{\mathrm{k}} p\right]$, =

In handy toy language, we can introduce the following label conditions for any $(k=0,1, \cdots)$.

(1) $[p]_{\mathbf{k}}$ stands for the $\mathbf{k}$ variable toy notion with labels of the form $[x, \cdots, y / x \cdots y p \mathrm{~B}]$ for any non-repetitive series $x, \cdots, y, p$ of the length $\mathrm{k}+1$.

(2) $\subset_{k}$ stands for the toy notion with labels of the form $\left[p, q / \forall x \cdots y \frac{x \cdots y[p]_{k}}{x \cdots y[q]_{k}}\right]$ for any non-repetitive series $p, q, x, \cdots, y$ of the length $\mathrm{k}+2$.

(3) $\varepsilon_{\mathrm{k}}$ stands for the toy notion with labels of the form $\left[x, y / x \cdots x[y]_{\mathrm{k}}\right]$ for a non-repetitive series $x, y$ and a repetitive series $x, \cdots, x$ of the length $\mathrm{k}$.

(4) $\left[\varepsilon_{\mathrm{k}} p\right]$ stands for the toy notion with labels of the form $\left[x / x \varepsilon_{\mathrm{k}} p\right]$ for any $x$ distinct from $p$.

(5) = stands for the toy notion with labels of the form [x,y/xyE].

8.1.1. We prefer the forms $x \subset_{\mathrm{k}} y, x \varepsilon_{\mathrm{k}} y$, and $x=y$ to the standard forms $x y \subset_{\mathrm{k}}$, $x y \varepsilon_{k}$, and $x y=$, respectively. 
8.1.2. The followings are easily provable:

(1) $[p]_{\mathrm{k}}{ }^{\left[\varepsilon_{\mathrm{n}} q\right]}$ is similar to $[p]_{\mathrm{k}}$ for any $p$ and $q$ and for any $\mathrm{k}$ and $\mathrm{n} .(=0,1, \cdots)$

(2) $\varepsilon_{\mathrm{k}}{ }^{\left[\varepsilon_{\mathrm{n}} p\right]}$ is similar to $\varepsilon_{\mathrm{k}}$ for any $p$, and for any $\mathrm{k}$ and $\mathrm{n}(=0,1, \cdots)$

\subsection{Mini-universe notion}

Whenever we approve a proposition, we are apt to imagine a mini-universe where the proposition holds. The mini-universe notion seems rather vague in our real thinking. However, we admit

(1) Any object in any mini-universe is included in the mini-universe,

(2) Any part of an object in any mini-universe is in the mini-universe, anyway.

We introduce our mini-universe notion along this line.

8.2.1. $\mathrm{U}_{\mathrm{k}}$ stands for the toy notion with labels of the form

$$
\left[u / \wedge\left(\begin{array}{l}
\forall x \cdots y\left(x \cdots y[u]_{\mathrm{k}} \equiv \wedge\left(x \varepsilon_{\mathrm{k}} u, \cdots, y \varepsilon_{\mathrm{k}} u\right)\right) \\
\forall z \frac{z \varepsilon_{\mathrm{k}} u}{z \subset_{\mathrm{k}} u}, \forall x \cdots y s \cdots t \frac{s \subset_{\mathrm{k}} x, \cdots, t \subset_{\mathrm{k}} y, x \cdots y[u]_{\mathrm{k}}}{s \cdots t[u]_{\mathrm{k}}}
\end{array}\right)\right]
$$

for any $u$ distinct from $x, \cdots, y, z, s, \cdots, t$.

$u \mathrm{U}_{\mathrm{k}}$ reads " $u$ is a mini-universe with respect to $\mathrm{k} . "(\mathrm{k}=0,1, \cdots)$

8.2.2. The mini-universe notion $U_{k}$ with labels of the form $U_{k}$ has the following properties: $(\mathrm{k}=0,1, \cdots)$

(1) $\forall u x y \frac{u U_{\mathrm{k}}, x \varepsilon_{\mathrm{k}} y, y \varepsilon_{\mathrm{k}} u}{x \varepsilon_{\mathrm{k}} u}$

(2) $\forall u x y \frac{u U_{\mathrm{k}}, x \varepsilon_{\mathrm{k}} u}{\left(x \subset_{\mathrm{k}} y\right)^{\left[\varepsilon_{\mathrm{k}} u\right]} \equiv\left(x \subset_{\mathrm{k}} y\right)}$

(3) $\forall u z \frac{u U_{\mathrm{k}}, z \varepsilon_{\mathrm{k}} u}{\left(z U_{\mathrm{k}}\right)^{\left[\varepsilon_{\mathrm{k}} u\right]} \equiv z U_{k}}$

8.3. Mini-universe imagination principle

Whenever we approve any proposition, we are apt to imagine a mini-universe where the proposition holds.

8.3.1. We can describe our mini-universe imagination in the label sentence form

$$
\forall p \cdots q \frac{F}{\exists u\left(u U_{\mathbf{k}}, F^{\left[\varepsilon_{\mathrm{k}}{ }^{\prime]}\right)}\right.}
$$

for any non-repetitive series $p, \cdots, q$, any label sentece $F$, and any $u$ distinct from $p, \cdots, q$ and not occuring free in $F .(k=1,2, \cdots)$

We call the principle admitting all the toy propositions with labels of this form the mini-universe imagination principle.

\subsection{Toy abstraction principle}

Whenever we have any condition, we are apt to think of an object which represent the condition. However, it is well known that we can not admit this in 
general.

8.4.1. Just as the subset axiom in the usual set theory, we may assume representation of any condition on variable objects, which satisfy a condition represented, by an object.

8.4.2. We can describe this in the following label sentence form:

$$
\forall p \cdots q \exists c \forall x \cdots y\left(x \cdots y[c]_{k} \equiv \wedge\left(x \cdots y[q]_{k}, F\right)\right)
$$

for any non-repetitive series $p, \cdots, q$ of any length no shorter than 1 , any nonrepetitive series $x, \cdots, y$, any label sentence $F$, and any $c$ not occuring free in F. $(\mathrm{k}=1,2, \cdots)$

We call the priciple admitting all the toy propositions with labels of this form the toy abstraction principle.

8.5. We call handy toy language admitting

(1) $\forall x(x=x)$,

(2) $\forall p \cdots q x y \frac{x=y}{x[1 ! C] \equiv y[1 ! C]}$ for any non-repetitive series $p, \cdots, q, x, y$ of any length no shorter than 2 and any label condition of the form [1!C].

(3) Mini-universe imagination principle (See 8.3.1.),

(4) Toy abstraction principle (See 8.4.1.) imaginative toy language.

8.5.1. We are expecting that imaginative toy language would give a practical clue to understanding of our real activity of developing mathematical theories, and further, it may exert back influence upon our ordinary language for mathematical thinking.

Indeed, we can develop a really rich theory resembling set theory in various respects in any imaginative language, which we will write elsewhere in details.

8.6. Toy theories of mathematics

We are expecting to be able to formalize simple mathematical theories of these days as follows:

Any toy theory of mathematics has a limited number of toy propositions $\mathbf{P}$, $\cdots, \mathbf{Q}$ as its postulates. Any toy proposition $\mathbf{T}$ is regarded as a toy theorem of the toy theory if and only if $\mathbf{T}$ is deducible from $\mathbf{P}, \cdots, \mathbf{Q}$ imaginative toy language. 8.6.1. As am example of toy theories of this kind, let us take up a toy theory of natural numbers. As postulates of the toy theory, let us take up Peano's axioms except for the axiom of complete induction in label sentence forms as follows:

(N1) $\exists x(x 0) \quad(x 0$ is expected to mean " $x$ is zero")

(N2) $\forall x \exists y(y x \mathrm{~S}) \quad(y x \mathrm{~S}$ is expected to mean " $y$ is a successor of $x$ ")

(N3) $\forall s t x y \frac{t s S, y x S}{t=y \equiv x=s}$

(N4) $\forall x y(\supset(\wedge(x 0, x y \mathrm{~S})))$. 
Then, in imaginative language, we can introduce a toy notion with labels of the form $[1 ! M]$ standing for $\left.[m / \wedge((\mathrm{N} 1),(\mathrm{N} 2),(\mathrm{N} 3),(\mathrm{N} 4))))^{\left[\varepsilon_{2} m\right]}\right]$, and further, a toy notion with labels of the form $[1 ! N]$ for $\left[n / \wedge\left(n[1 ! M], \forall m \frac{m[1 ! M]}{n \subset_{1} m}\right)\right]$

We can prove in imaginative toy language

(1) $3 n(n[1 ! N])$,

(2) $\forall n \frac{n[1 ! N], \forall x \frac{x 0}{x[1 ! C]}, \forall x y \frac{y x S, x[1 ! C]}{y[1 ! C]}}{\forall z \frac{z \varepsilon_{1} m}{z[1 ! C]}}$ for any label condition of the form $[1-C]$.

Thus, we can see that there is a domain ( $n$ such that $n[1 ! N]$ ) where all the Peano axioms including the complete induction holds.

\section{References}

[1] K. Ono, Frame-works of thoughts (in Japanese), I, J. of Jap. Ass. for Phil of Sci. 58 (1981) 125-135, II (To be published).

[2] $\stackrel{2}{-}$, On a practical way of describing formal deductions, Nagoya Math. J., 21 (1962), 115-121.

[3] - , On universal character of the primitive logic, Nagoya Math. J., 27 (1966), 331-353.

[4] $\stackrel{331-353 .}{-}$, Reduction of logics to the primitive logic, J. Math. Soc. Japan, 19-3 (1967), 384-398. 